\title{
On the puzzling distribution of cholesterol in the plasma membrane
}

\author{
H. Giang and M. Schick \\ Department of Physics, University of Washington,Seattle, WA 98195
}

November 29, 2015 


\begin{abstract}
The distribution of cholesterol between the two leaves of the plasma membrane in mammalian cells presents a conundrum; given cholesterol's known affinity for sphingomyelin, which resides predominantly in the exoplasmic leaf, why is it that experiment finds a majority of the cholesterol in the cytoplasmic leaf? This chapter reviews a recently proposed solution to this puzzle.
\end{abstract}




\section{Introduction}

Given the importance of cholesterol in the cell, one would have thought that its distribution between the two leaves of the plasma membrane would not only be well-known, but also well-understood. There is a certain amount of agreement now on what the distribution is, but little understanding on why it is what it appears to be. To clarify the problem, one should recall some basic information. First it is well known that cholesterol can translocate rapidly between the two leaves of the plasma membrane (1-3). Thus one would assume that it reaches an equilibrium distribution in which its chemical potentials in the two leaves are the same. Second the interaction between cholesterol and sphingomyelin (SM) is known to be particularly favorable (4) being due to that between the rigid rings of the former and the saturated chains of the latter $(5,6)$. Third, almost all of the sphingomyelin is in the outer, exoplasmic, leaf of the plasma membrane (7). It would then seem to follow inexorably that the majority of cholesterol would be found in the outer leaf; its chemical potential there would be negative due to the favorable interaction with SM, while its chemical potential in the inner leaf would be equally negative due to its entropy at low-concentration. That this conclusion does follow from the chain of reasoning finds support from molecular dynamics simulations $(8,9)$.

While the reasoning may be impeccable, the conclusion is not borne out by several experiments on human erythrocytes $(2,10-14)$, chinese hamster ovaries, (17), and synaptic plasma membranes $(15,16)$. All uniformly agree that cholesterol is not found predominantly in the exoplasmic leaf. A similar uniformity does not exist as to what the distribution of cholesterol actually is. Some report that cholesterol is rather evenly divided between the two leaves $(2,10-12)$, while others find that it is predominantly found in the inner, cytoplasmic, leaf (13-17). The experiments do not measure native cholesterol directly, but employ various cholesterol analogs labeled in some way. Thus the difference in measured distribution may be due to the different analogs employed. Nonetheless it is clear that cholesterol is is not found predominantly in the outer leaf where the sphingomyelin is, hence something must be drawing it to the inner leaf. But what? That is the puzzle.

We recently proposed a solution to it as follows (18): Phosphatidylethanolamine is one of the major components of the inner leaf of the plasma membrane comprising some $25 \%$ of the phospholipids there (7). Because of its small head group, phosphatidyethanolamine (PE) has a tendency to form inverted hexagonal phases in water. For palmitoyloleoylphosphatidylcholine, that phase becomes stable around a temperature of $70^{\circ} \mathrm{C}$. Thus, when it is 
confined to a bilayer, there must be some bending energy penalty for this confinement. We proposed that cholesterol could relieve in two ways this bending energy penalty by going to the cytoplasmic leaf. First of all, its presence would simply dilute that of the PE. Of course that is true of any other lipid as well, but cholesterol is the only one which translocates between leaves. Secondly it is well known that cholesterol tends to order the tails of lipids. Hence it might well reduce the spontaneous curvature of the PE lipids and thereby further reduce the bending energy penalty of confining $\mathrm{PE}$ to a bilayer. If the above reasoning were correct, then one would expect that the addition of cholesterol to a system of $\mathrm{PE}$ and water would stabilize the lamellar phase of $\mathrm{PE}$ and therefore increase the transition temperature from the lamellar phase to the inverted hexagonal phase. That experiment has been done (19). On the initial addition of cholesterol to a PE and water system, the transition temperature decreases; that is, the invertedhexagonal phase is stabilized. Presumably this is due to the cholesterol filling the energetically expensive interstial voids between cylinders. But with further addition of cholesterol to concentrations which are comparable to those in the plasma membrane, the transition temperature does indeed increase. Thus sufficient cholesterol does stabilize the lamellar phase of PE in water.

In order to make these qualitative ideas quantitative, we considered a very simple and standard model for the free energy of the system, namely regular solution theory. We take the system to consist of two leaves. There are $N_{S M}$ molecules of SM, $N_{C_{o}}$ of cholesterol, and $N_{P C}$ molecules of phosphatidylcholine (PC) in the outer layer, and $N_{P E}$ molecules of PE, $N_{C_{i}}$ molecules of cholesterol, and $N_{P S}$ molecules of PS phosphatidylserine (PS) in the inner layer. The area per lipid of all lipids is taken to be $a=0.7 \mathrm{~nm}^{2}$ except for cholesterol which is taken to be $a_{c}=0.4 \mathrm{~nm}^{2}$. We require the areas of each leaf to be the same,

$$
\left(N_{S M}+N_{P C}\right) a+N_{C_{o}} a_{c}=\left(N_{P E}+N_{P S}\right) a+N_{C_{i}} a_{c}=A .
$$

It is convenient to define the mol fractions of the components in the outer leaf, $y_{S M}=N_{S M} /\left(N_{S M}+N_{C_{o}}+N_{P C}\right)$ etc. and similarly for the mol fractions in the inner leaf. In terms of these mol fractions, the regular 
solution free energy can be written

$$
\begin{aligned}
F_{b i}= & N_{o} f_{o}\left(y_{S M}, y_{P C}, y_{C_{o}}, T\right)+N_{i} f_{i}\left(y_{P E}, y_{P S}, y_{C_{i}}, T\right), \\
f_{i}= & 6 \epsilon_{P S, P E} y_{P S} y_{P E}+6 \epsilon_{P S, C} y_{P S} y_{C_{i}}+6 \epsilon_{P E, C} y_{P E} y_{C_{i}}+ \\
& k_{B} T\left(y_{P S} \ln y_{P S}+y_{P E} \ln y_{P E}+y_{C_{i}} \ln y_{C_{i}}\right), \\
f_{o}= & 6 \epsilon_{S M, P C} y_{S M} y_{P C}+6 \epsilon_{S M, C} y_{S M} y_{C_{o}}+6 \epsilon_{P C, C} y_{P C} y_{C_{o}}+ \\
& k_{B} T\left(y_{S M} \ln y_{S M}+y_{P C} \ln y_{P C}+y_{C_{o}} \ln y_{C_{o}}\right) .
\end{aligned}
$$

where $N_{o}$ and $N_{i}$ are the total numbers of molecules in the outer and inner leaves respectively, and $\epsilon_{P S, P E}$ is the free energy of interaction between the PS and PE. We have assumed an average of six nearest-neighbor interactions per molecule. These interactions are estimated from experiment $(18,20)$. From the free energy, the chemical potential of cholesterol in the inner and outer leaves can be obtained. The six unknown mol fractions are then obtained from the following six conditions: the chemical potentials of cholesterol in both leaves are equal to one another, the mol fractions in the outer leaf sum to unity, as do the mol fractions in the inner leaf, the ratios of the mol fractions of SM to PC in the outer leaf are set to be 1.1 and of PS to PE in the inner leaf to be 0.52 , values essentially given by experiment. Finally the total mol fraction of cholesterol in the system is taken to be fixed at its experimental value, 0.4 (21). Once the mol fractions are calculated, it is straightforward to obtain the fraction of the total cholesterol which is in each leaf.

If we follow this program with the above simple model, we find that only $25 \%$ of the total cholesterol is in the inner leaf. This is not surprising, and reflects the reasoning we laid out in the Introduction: cholesterol interacts favorably with SM, almost all the SM is in the outer leaf, hence that is where most of the cholesterol should be. So the simple model vindicates the impeccable reasoning, and confirms that it is missing something essential. Consequently we add the bending energy to it.

The bending energy can be written as

$$
F_{b}=\int d^{2} r \frac{\kappa}{2}\left(H(r)-H_{0}\right)^{2}
$$

where $\kappa$ is the bending modulus of the bilayer, $H(r)$ is the actual local curvature of the system, and $H_{0}$ is the preferred, or spontaneous, curvature of the membrane due to its composition. This expression assumes that there is no intrinsic difference in energy to bend the bilayer toward the cytoplasmic side or the exoplasmic side. Because we take the membrane to be flat, 
$H(r)=0$. We assume that the local spontaneous curvature of the bilayer is a weighted sum of the spontaneous curvatures of its components. However the only component in the system which has a spontaneous curvature of significant magnitude is $\mathrm{PE}(22)$, for which $H_{P E}=-0.316 \mathrm{~nm}^{-1}$. Thus this energy reduces to

$$
\begin{aligned}
F_{b} & =A \frac{1}{2} \kappa y_{P E}^{2} H_{P E}^{2} \\
& =\frac{1}{2}\left[N_{i}+N_{o}-\left(1-\left(a_{c} / a\right)\right)\left(N_{C_{o}}+N_{C_{i}}\right)\right] \frac{1}{2} a \kappa y_{P E}^{2} H_{P E}^{2} .
\end{aligned}
$$

Note that this term is quadratic in the mol fraction of $\mathrm{PE}$ and is positive; i.e. the bending energy is a cost. In this way, the bending energy acts as a repulsive interaction between the $\mathrm{PE}$ molecules or, equivalently, an attractive interaction between $\mathrm{PE}$ and all of the other kinds of molecules. The cholesterol, however, is the only lipid in the outer leaf that can readily respond to this attraction by translocating to the inner leaf where it dilutes the PE. Because the majority of experiments have been done on erythrocytes, we take the bending modulus to be $\kappa=44 k_{B} T$, that obtained from measurement on red blood cells (23), and repeat our procedure. We find that the fraction of the total cholesterol in the inner leaf has increased from $25 \%$ to $38.6 \%$.

We now implement the idea that cholesterol reduces the spontaneous curvature of $\mathrm{PE}$, presumably by ordering its tails. In other words, we take the spontaneous curvature of $\mathrm{PE}$ to be cholesterol-dependent. To model this dependence, we imitate the effect of cholesterol on the temperature of transition from lamellar to inverted-hexagonal phase of PE in water (19). In particular, we will require that the spontaneous curvature begin to decrease when the mol fraction of cholesterol in the inner leaf is approximately 0.3 , and to decrease rapidly when the mol fraction is about 0.4 . We choose the form

$$
H_{P E}\left(y_{C_{i}}\right)=H_{P E}^{0}-B \frac{y_{C_{i}}}{y_{\min }}+\frac{B}{\lambda}\left(\frac{y_{C_{i}}}{y_{\min }}\right)^{\lambda},
$$

with $B=0.05, y_{\min }=0.3$, and $\lambda=8$. This form is shown in Fig. 1 for several choices of $\lambda$.

With this choice of a cholesterol-dependent spontaneous curvature, we find that $58 \%$ of the total cholesterol is in the inner leaf. Thus the mechanism we propose does lead to the conclusion that the majority of cholesterol in the plasma membrane should be found in the inner leaf, the reason being 


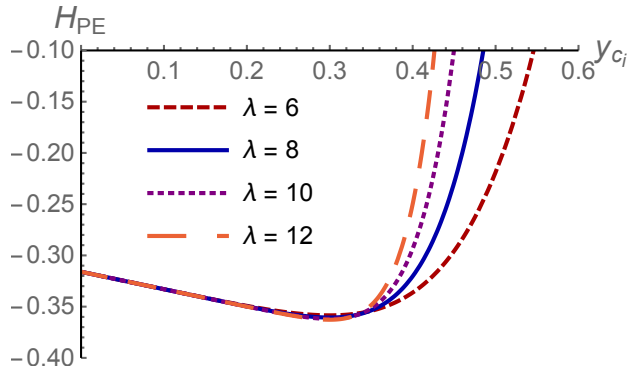

Figure 1: Spontaneous curvature of $\mathrm{PE}$ in the presence of cholesterol as modeled by Eq.(2) for four values of $\lambda$

that that is where the PE is. The mol fractions of the three components in the outer leaf are $y_{S M}=0.34, y_{P C}=0.31, y_{C_{o}}=0.35$ while those of the inner leaf are $y_{P S}=0.19, y_{P E}=0.36$, and $y_{C_{i}}=0.45$.

Because our mechanism depends upon the bending energy, and the bending moduli of the bilayers enclosing different vesicles within the cell are presumably different, it is of interest to examine how the fraction of cholesterol depends upon the bending modulus, all other things being equal. This is shown in Fig. 2

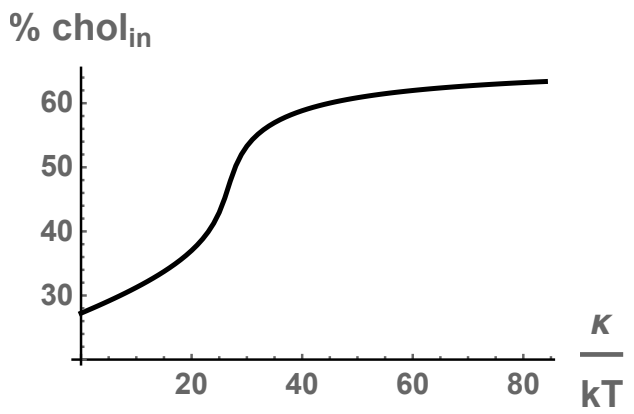

Figure 2: Percent of cholesterol in the inner leaf as a function of the bending modulus $\kappa$.

Our mechanism also depends upon the total amount of cholesterol in the membrane which is only large in the plasma membrane and late-stage endosomes (24). Thus it is of interest to see how the fraction of cholesterol in the inner leaf depends upon the total amount of cholesterol in the membrane. That is shown in Fig. 3. One sees that if there is not much cholesterol and if there were SM in the outer leaf, little cholesterol would be in the inner 
leaf. The fraction of cholesterol in the inner leaf increases with the total fraction of cholesterol and must attain the value of $1 / 2$ were the membrane to be completely cholesterol. The interesting result is that the fraction of cholesterol in the inner leaf exceeds $1 / 2$ for values of the total cholesterol content typical of human erythrocytes (21).

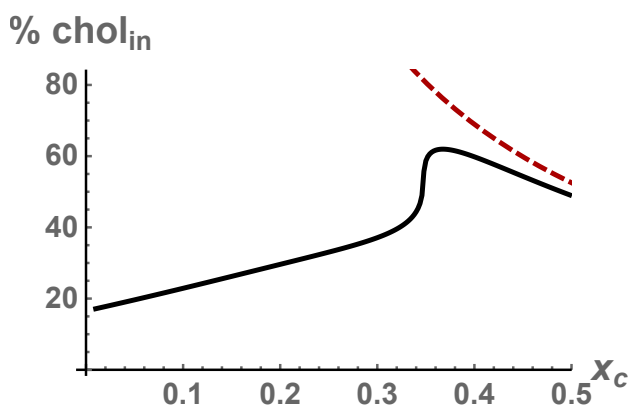

Figure 3: Percent of total cholesterol in the inner leaf as a function of the total cholesterol mol fraction $x_{C}$. The dashed line shows the solubility limit of cholesterol.

\section{Conclusion}

We have proposed a way to understand the experimental observations that the majority of the cholesterol in the mammalian plasma membrane is found in the inner, cytoplasmic, leaf; it is because that is where then phosphatidylethanolamine is. Its presence there comes at the cost of a bending energy penalty which the presence of cholesterol can relieve, both by diluting the $\mathrm{PE}$ and by reducing its spontaneous curvature. This idea ties together the cholesterol distribution in the plasma membrane with completely independent experiments which show that sufficient amounts of cholesterol stabilize the lamellar phase of $\mathrm{PE}$ in water relative to the inverted-hexagonal phase $(19,25)$. There are additional effects which could either decrease or increase the fraction of cholesterol in the inner leaf. Among the former is the fact that cholesterol is not very soluble in polyunsaturated fatty acids (26), and such chains are found predominantly in the inner leaf. As for the latter, it has been suggested that the presence of SM in the outer leaf with very long chains that reach into the inner leaf could attract the cholesterol there (27). In addition, we have utilized the simple form for the bending energy of Eq.(1) and have assumed that the spontaneous curvature of a mixture of 
lipids is a weighted sum of their spontaneous curvatures,. This is a reasonable assumption, but one which must be checked (28). Further the form of the bending energy assumes that bending toward the cytosol or away from it are energetically equivalent. Because of the cytoskeleton, this may not be the case. Were the bending energy proportional to the first power of the PE concentration rather than to the second as we have assumed, the fraction of cholesterol in the inner leaf would certainly be greater than what we have obtained here.

Be that as it may, we believe that the mechanism that we have proposed goes a long way in resolving the puzzling distribution of cholesterol in the plasma membrane.

\section{Acknowledgments}

We wish to thank Paulo Almeida, William Clay and Josh Zimmerberg, and Richard Epand for stimulating conversations, This work was supported by the National Science Foundation under grant No. DMR-1203282. 


\section{References}

1. Lange, Y., J. Dolde, and T. Steck. 1981. The rate of transmembrane movement of cholesterol in the human erythrocyte. J. Biol. Chem. 256:5321-5323.

2. Muller, P., and A. Hermann. 2002. Rapid transbilayer movement of spin-labeled steroids in human erythrocytes and in liposomes. Biophys. J. 82:1418-1428.

3. Steck, T., and Y. Lange. 2002. Probing red blood cell membrane cholesterol movement with cyclodextrin. Biophys. J 83:2118-2125.

4. Niu, S.-L., and B. Litman. 2002. Determination of membrane cholesterol partition coefficient using a lipid vesicle-cyclodextrin binary system: Effect of phospholipid acyl chain unsaturation and headgroup composition. Biophys. J. 83:3408-3415.

5. Epand, R., and R. F. Epand. 2004. Non-raft forming sphingomyelincholesterol mixtures. Chem. Phys. Lipids 132:37-46.

6. Zheng, L., C. McQuaw, A.G.Ewing, and N. Winograd. 2007. Sphingomyelin/phosphatidylcholine and cholesterol interactions studied by imaging mass spectroscopy. J. Am. Chem. Soc. 129:15730-15731.

7. Devaux, P. 1991. Static and dynamic lipid asymmetry in cell membranes. Biochemistry 30:1163-1173.

8. Perlmutter, J. D., and J. N. Sachs. 2011. Interleaflet interaction and asymmetry in phase separated lipid bilayers: Molecular dynamics simulations. JACS 133:6563-6577.

9. Polley, A., S. Vemparala, and M. Rao. 2012. Atomistic simulations of a multicomponent asymmetric lipid bilayer. J. Phys. Chem. B 116:1340313410 .

10. Blau, L., and R. Bittman. 1978. Cholesterol distribution between the two leaves of the lipid bilayer of the human erythrocyte; ghost membranes. J. Biol. Chem. 253:8366-8368.

11. Lange, Y., and J. M. Slayton. 1982. Interaction of cholesterol and lysophosphatidylcholine in determining red cell shape. J. Lipid Res. 23:1121-1127. 
12. Lange, Y. 1984. The dynamics of erythrocyte membrane cholesterol. Prog. Clin. Biol. Res.. 159:137-151.

13. Brasaemle, D., A. Robertson, and A. Attie. 1988. Transbilayer movement of cholesterol in the human erythrocyte membrane. J. Lipid Res. 29:481-489.

14. Schroeder, F., G. Nemecz, W. G. Wood, C. Joiner, G. Morrot, M. Ayraut-Jarrier, and P. Devaux. 1991. Transmembrane distribution of sterol in the human erythrocyte. Biochimica Et Biophysica Acta 1066:183-192.

15. Wood, W., F. Schroeder, L. Hogy, A. Rao, and G. Nemecz. 1990. Asymmetric distribution of a fluorescent sterol in synaptic plasma membrane: effects of chronic ethanol consumption. Biochim. Biophys. Acta 1025:243-246.

16. Igbavboa, U., N. Avdulov, F. Schroeder, and W. Wood. 1996. Increasing age alters transbilayer fluidity and cholesterol asymmetry in synaptic plasma membranes of mice. J Neurochem 66.

17. Mondal, M., B. Mesmin, S. Mukherjee, and F. Maxfield. 2009. Sterols are mainly in the cytoplasmic leaflet of the plasma membrane and the endocytic recycling compartment of CHO cells. Mol. Biol. Cell 20:581588 .

18. Giang, H., and M. Schick. 2014. How cholesterol could be drawn to the cytoplasmic leaf of the plasma membrane by phosphatidylethanolamine. Biophys. J. 107:2337-2344.

19. Epand, R., and R. Bottega. 1987. Modulation of the phase transition behavior of phosphatidylethanolamine by cholesterol and oxysterols. Biochemistry 26:1820-1825.

20. Almeida, P. 2009. Thermodynamics of lipid interactions in complex bilayers. Biochim. Biophys. Acta 1788:72-85.

21. van Meer, G. 2011. Dynamic transbilayer lipid asymmetry. Cold Spring Harb Perspect Biol 3:1-11.

22. Kollmitzer, B., P. Heftberger, M. Rappolt, and G. Pabst. 2013. Monolayer spontaneous curvature of raft-forming membrane lipids. Soft Matter 9:10877-10884. 
23. Evans, E. 1983. Bending elastic modulus of red blood cell membrane derived from buckling instability in micropipet aspiration tests. Biophys. J. 43:27-30.

24. van Meer, G., D. Voelker, and G. W. Feigenson. 2008. Membrane lipids: where they are and how do they behave? Nat. Rev. Mol. Cell Biol 112:112-124.

25. Takahashi, H., K. Sinoda, and I. Hatta. 1996. Effects of cholesterol on the lamellar and the inverted hexagonal phases of dielaidoylphosphatidylethanolamine. Biochim. Biophys. Acta. 1289:209-216.

26. Brzustowicz, M., V. Cherezov, M. Zerouga, M. Caffrey, and S. Wassail. 2002. Controlling membrane cholesterol content. a role for polyunsaturated (docosahexaenoate) phospholipids. Biochemistry. 41:12509-12519.

27. Courtney, K., C. Zhang, and X. Zhai. 2015. Long acyl chain sphingolipids govern visible microdomains and cholesterol in both model and plasma membranes. Biophysi. J. 108:32a.

28. Sodt, A., R. Venable, E. Lyman, and R. Pastor. 2015. Lipid-lipid interactions determine the membrane spontaneous curvature. Biophys. J 108:181a. 